\title{
The Epsilon Probability Distribution and its Application in Reliability Theory
}

\author{
József Dombi ${ }^{1}$, Tamás Jónás ${ }^{2}$, Zsuzsanna Eszter Tóth ${ }^{2}$ \\ ${ }^{1}$ Department of Computer Algorithms and Artificial Intelligence \\ University of Szeged \\ Árpád tér 2, H-6720 Szeged, Hungary \\ dombi@inf.u-szeged.hu \\ ${ }^{2}$ Institute of Business Economics \\ Eötvös Loránd University \\ Egyetem tér 1-3, H-1053 Budapest, Hungary \\ jonas@gti.elte.hu, tothzs@gti.elte.hu
}

\begin{abstract}
This paper elaborates a new probability distribution, namely, the epsilon probability distribution with implications for reliability theory and management. This probability distribution is founded on the so-called epsilon function that is introduced here. It is also shown that the asymptotic epsilon function is just an exponential function. The properties of this probability distribution suggest that it may serve as a viable alternative to the exponential probability distribution. As the epsilon probability distribution function is a power function, it is more convenient than the exponential probability distribution function from a computational point of view. The main findings and a practical example indicate that the new probability distribution can be utilized to describe the probability distribution of the time to first failure random variable both in the second and third phases of the hazard function.
\end{abstract}

Keywords: exponential probability distribution; epsilon probability distribution; hazard function, failure rate modeling

\section{Introduction}

The exponential probability distribution as one of the key distributions in the theory and practice of reliability management [3] [4] plays a significant role in analyzing many data sets obtained from life-tests, and in the use of order statistics. This distribution also appears frequently in lifetime and reaction time studies. It has several remarkable statistical properties, most notably, its characterization through the lack of memory property. Furthermore, it provides mathematical traceability [5]. Consequently, there is extensive literature on the theory and applications of the exponential distribution from the 1930s (e.g. [6], [7], [8]). Weibull [9] considered an 
extension of the exponential distribution referred to as Weibull distributions, including the exponential distribution as a special case where the shape parameter equals one. Davis [10] also discussed the analysis of failure rate data using the exponential distribution. Characterizations of the exponential distribution originate from mathematicians [11] [12]. Some researchers derived characterizations of the exponential distribution which are modifications of characterizations of the normal distribution [13] [14]. Since then, the characterization results for the exponential distribution have been paid significant attention [15].

In reliability analysis the negative exponential model provides simple, closed-form solutions to many problems [16]. Weibull's classic generalization is usually applied for modelling systems with monotone failure rates [9]. However, according to data in reliability analysis, the operation of a device population can generally be divided into three distinct periods called infancy, useful life, and wear-out periods with each region corresponding to a specific type of failure. This hazard-rate curve, which typically maps the failure rate versus time, has been verified by experience for many types of products. In reliability theory this bathtub-shape is widely used to describe the failure patterns of different products. The relevant literature regarding the bathtub curve is quite diverse. An overview of bathtub-shaped failure rate distributions are provided by [17] and [18]. The exponential distribution is widely applied for modeling the bathtub-shaped failure rate, mainly the useful life period [19], [20], [22].

Models which allow only monotone failure rates might not be appropriate or adequate for modeling the populations that give rise to such data. There have been several attempts to address the need for a family of distributions which allow flexibility in modeling. In this paper, a new probability distribution, namely, the epsilon probability distribution is introduced and its application in reliability theory is discussed. This novel probability distribution is founded on the so-called epsilon function which, just like the exponential function, may be deduced from the $n^{\text {th }}$ order epsilon differential equation. The solution of the zero order epsilon differential equation is the exponential function, while the solution of the first order epsilon differential equation is the epsilon function. The epsilon probability distribution has two parameters; namely a $\lambda$ parameter that has the same meaning as the $\lambda$ parameter in the exponential probability distribution, and a $d$ parameter that determines the domain $(0, d)$ where the epsilon probability distribution is defined $(d>0)$. Next, it is shown that the asymptotic epsilon probability distribution is just the exponential probability distribution, which means in practice that the exponential probability distribution with a parameter $\lambda$ can be substituted by the epsilon probability distribution that has parameters $\lambda$ and $d$. Besides the connection between the epsilon and the exponential functions, the relationship between continuous-valued logic and the epsilon function is also highlighted. Namely, the generator function of the Dombi operators [20] may be considered as a special case of the epsilon function.

The remaining part of the paper is organized as follows. In Section 2 the epsilon function, its basic properties and its connection with the exponential function are introduced. In Section 3 we define the epsilon probability distribution and introduce its asymptotic properties. In Section 4 its application in reliability theory is demon- 
strated through a practical example. Finally, key conclusions are drawn related to the new probability distribution.

\section{Epsilon Function}

Here we introduce the $n^{\text {th }}$ order epsilon differential equation.

Definition 1. We define the $n^{\text {th }}$ order epsilon differential equation as

$\frac{\mathrm{d} f(x)}{\mathrm{d} x}=\lambda\left(\frac{d^{2}}{d^{2}-x^{2}}\right)^{n} f(x)$,

where $\lambda \in \mathbb{R}, \lambda \neq 0, d \in \mathbb{R}, d>0, x \in \mathbb{R}, x \neq d, f(x)>0, n \in \mathbb{N}$.

Lemma 1. If $n=0$ and $x \in \mathbb{R}$, then the solution of the $n^{\text {th }}$ order epsilon differential equation is

$f(x)=\mathrm{e}^{\lambda x+C}$,

where $C \in \mathbb{R}$.

Proof. If $n=0$, then the differential equation in (1) may be written as

$\frac{\mathrm{d} f(x)}{\mathrm{d} x}=\lambda f(x)$.

Separating the variables in (3) results in

$\int \frac{1}{f(x)} \mathrm{d} f(x)=\lambda \int \mathrm{d} x$.

When we integrate both sides of this equation, we get

$\ln |f(x)|=\lambda x+C$,

and utilizing the fact, that $f(x)>0$ means that

$f(x)=\mathrm{e}^{\lambda x+C}$,

where $C \in \mathbb{R}$.

Note that if we wish $f(x)$ to satisfy the condition $f(0)=1$, then parameter $C$ in (6) needs to be set to 0 .

Lemma 2. If $n=1$ and $x \in(-d,+d)$, then the solution of the $n^{\text {th }}$ order epsilon differential equation is

$f(x)=C\left(\frac{x+d}{d-x}\right)^{\lambda \frac{d}{2}}$,

where $C>0$. 
Proof. If $n=1$, then the differential equation in (1) has the form

$\frac{\mathrm{d} f(x)}{\mathrm{d} x}=\lambda \frac{d^{2}}{d^{2}-x^{2}} f(x)$.

This equation may be written as

$\frac{\mathrm{d} f(x)}{\mathrm{d} x}=\lambda \frac{d^{2}}{(d+x)(d-x)} f(x)$.

Since

$\frac{1}{(d+x)(d-x)}=\frac{1}{2 d}\left(\frac{1}{x+d}+\frac{1}{d-x}\right)$,

Equation (9) may be written as

$\frac{\mathrm{d} f(x)}{\mathrm{d} x}=\lambda \frac{d}{2}\left(\frac{1}{x+d}+\frac{1}{d-x}\right) f(x)$,

and separating the variables in (11) results in

$\int \frac{1}{f(x)} \mathrm{d} f(x)=\lambda \frac{d}{2} \int\left(\frac{1}{x+d}+\frac{1}{d-x}\right) \mathrm{d} x$.

After integrating both sides of this equation, we get

$\ln |f(x)|=\lambda \frac{d}{2} \ln |x+d|-\lambda \frac{d}{2} \ln |d-x|+\ln C$,

where $C>0$. Utilizing the fact, that $x \in(-d,+d)$ and $f(x)>0$ means that

$f(x)=C\left(\frac{x+d}{d-x}\right)^{\lambda \frac{d}{2}}$.

Note that if we wish $f(x)$ to satisfy the condition $f(0)=1$, then parameter $C$ in (14) needs to be set to 1 . In this case, we call the solution of the first order epsilon differential equation the epsilon function.

Definition 2. The epsilon function $\varepsilon_{\lambda, d}(x)$ is given by

$\varepsilon_{\lambda, d}(x)=\left(\frac{x+d}{d-x}\right)^{\lambda \frac{d}{2}}$,

where $\lambda \in \mathbb{R}, \lambda \neq 0, d \in \mathbb{R}, d>0, x \in(-d,+d)$.

\subsection{Some Basic Properties of the Epsilon Function}

Here, we state the most important properties of the epsilon function; namely, continuity, monotonity, limits and convexity. 
Continuity. $\varepsilon_{\lambda, d}(x)$ is a continuous function in $(-d,+d)$.

\section{Monotonicity.}

- If $\lambda>0$, then $\varepsilon_{\lambda, d}(x)$ is strictly monotonously increasing

- If $\lambda<0$, then $\varepsilon_{\lambda, d}(x)$ is strictly monotonously decreasing

- If $\lambda=0$, then $\varepsilon_{\lambda, d}(x)$ has a constant value of 1

in the interval $(-d,+d)$.

\section{Limits.}

$\lim _{x \rightarrow-d^{+}} \varepsilon_{\lambda, d}(x)= \begin{cases}0, & \text { if } \lambda>0 \\ \infty, & \text { if } \lambda<0\end{cases}$

$\lim _{x \rightarrow+d^{-}} \varepsilon_{\lambda, d}(x)= \begin{cases}\infty, & \text { if } \lambda>0 \\ 0, & \text { if } \lambda<0 .\end{cases}$

Note that if $\lambda>0$, then $\varepsilon_{\lambda, d}(-d)=0$, and if $\lambda<0$, then $\varepsilon_{\lambda, d}(d)=0$.

Convexity. The second derivative of $\varepsilon_{\lambda, d}(x)$ is

$\frac{\mathrm{d}^{2} \varepsilon_{\lambda, d}(x)}{\mathrm{d} x^{2}}=\lambda \frac{d^{2}}{\left(d^{2}-x^{2}\right)^{2}} \varepsilon_{\lambda, d}(x)\left(2 x+\lambda d^{2}\right)$,

whose sign depends on $\lambda$ and $2 x+\lambda d^{2}$. Thus,

- if $\lambda>0$ and $x<-\frac{\lambda d^{2}}{2}$, then $\varepsilon_{\lambda, d}(x)$ is concave,

- if $\lambda>0$ and $x>-\frac{\lambda d^{2}}{2}$, then $\varepsilon_{\lambda, d}(x)$ is convex,

- if $\lambda<0$ and $x<-\frac{\lambda d^{2}}{2}$, then $\varepsilon_{\lambda, d}(x)$ is convex,

- if $\lambda<0$ and $x>-\frac{\lambda d^{2}}{2}$, then $\varepsilon_{\lambda, d}(x)$ is concave.

That is, $\varepsilon_{\lambda, d}(x)$ has a single inflection point at $-\frac{\lambda d^{2}}{2}$. Notice that $-\frac{\lambda d^{2}}{2}$ lies in the interval $(-d, d)$, only if $|\lambda| d<2$. Hence, $\varepsilon_{\lambda, d}(x)$ is strictly convex in $(-d, d)$, if $|\lambda| d \geq 2$.

Figure 1 shows two examples of the epsilon function curve with positive and negative $\lambda$ parameter values.

\subsection{Connection with the Exponential Function}

Lemma 1 and Lemma 2 suggest an important connection between the exponential function and the epsilon function. Namely, the solution of the zero order epsilon differential equation is the exponential function, while the solution of the first order 


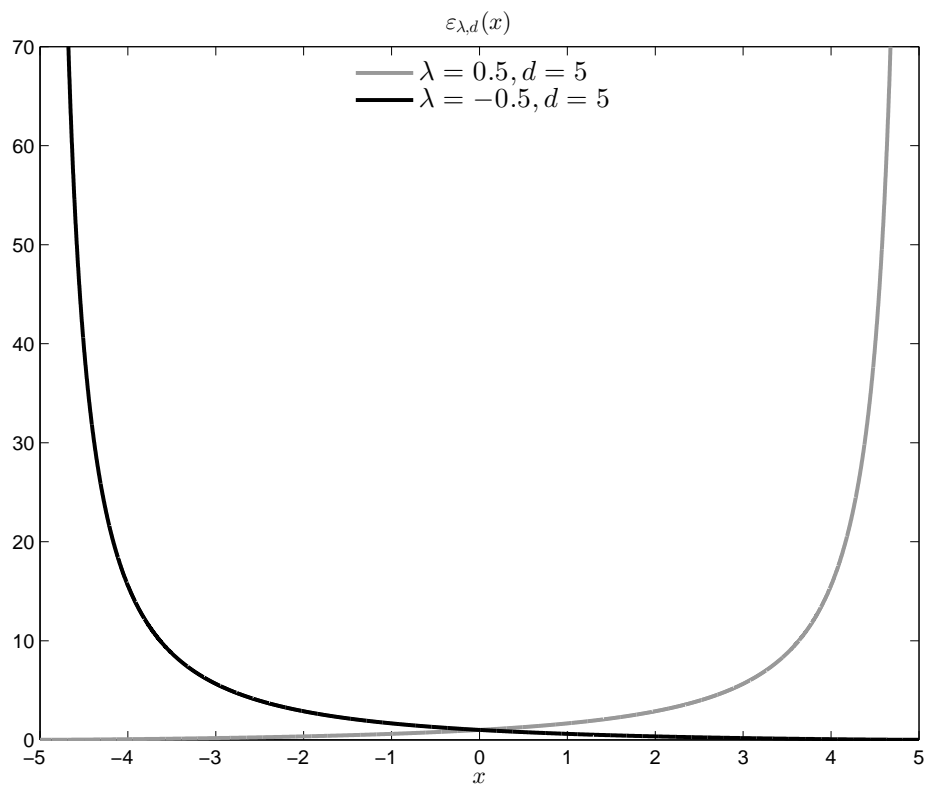

Figure 1

Examples of epsilon function curves

epsilon differential equation is the epsilon function. Table 1 summarizes how the exponential function and the epsilon function can be derived from the the $n^{\text {th }}$ order epsilon differential equation.

Table 1

The exponential and epsilon functions derived from the $n^{\text {th }}$ order epsilon differential equation

\begin{tabular}{lllll} 
Domain & $n$ & Condition & $C$ & $f(x)$ \\
\hline$(-\infty, \infty)$ & 0 & $f(0)=1$ & 0 & $\mathrm{e}^{\lambda x}$ \\
$(-d,+d)$ & 1 & $f(0)=1$ & 1 & $\varepsilon_{\lambda, d}(x)$ \\
\hline
\end{tabular}

Beyond the fact that both the exponential and the epsilon functions can be derived from the $n^{\text {th }}$ order epsilon differential equation, the following theorem highlights an additional connection between these two functions.

Theorem 1. For any $x \in(-d,+d)$, if $d \rightarrow \infty$, then

$\varepsilon_{\lambda, d}(x) \rightarrow \mathrm{e}^{\lambda x}$ 
Proof. Let $x$ have a fixed value, $x \in(-d,+d)$.

$$
\begin{aligned}
& \lim _{d \rightarrow \infty} \varepsilon_{\lambda, d}(x)=\lim _{d \rightarrow \infty}\left(\frac{x+d}{d-x}\right)^{\lambda \frac{d}{2}}=\lim _{d \rightarrow \infty}\left(\left(\frac{d-x+2 x}{d-x}\right)^{d}\right)^{\frac{\lambda}{2}}= \\
& =\lim _{d \rightarrow \infty}\left(\left(1+\frac{2 x}{d-x}\right)^{d}\right)^{\frac{\lambda}{2}} .
\end{aligned}
$$

Since $x$ is fixed, if $d \rightarrow \infty$, then $\Delta=d-x \rightarrow \infty$ and so the previous equation can be continued as follows.

$$
\begin{aligned}
& \lim _{d \rightarrow \infty}\left(\left(1+\frac{2 x}{d-x}\right)^{d}\right)^{\frac{\lambda}{2}}=\lim _{\Delta \rightarrow \infty}\left(\left(1+\frac{2 x}{\Delta}\right)^{\Delta+x}\right)^{\frac{\lambda}{2}}= \\
& =\left(\lim _{\Delta \rightarrow \infty}\left(1+\frac{2 x}{\Delta}\right)^{\Delta} \lim _{\Delta \rightarrow \infty}\left(1+\frac{2 x}{\Delta}\right)^{x}\right)^{\frac{\lambda}{2}}=\left(\mathrm{e}^{2 x}\right)^{\frac{\lambda}{2}} \cdot 1^{\frac{\lambda}{2}}=\mathrm{e}^{\lambda x} .
\end{aligned}
$$

Based on Theorem 1, we may state that with respect to parameter $d(d \rightarrow \infty)$, the asymptotic epsilon function is just the exponential function. Actually, if $x \ll d$, then $\varepsilon_{\lambda, d} \approx \mathrm{e}^{\lambda x}$; that is, if $d$ is sufficiently large, then the epsilon function suitably approximates the exponential function.

\subsection{Connection with Dombi Operators in Continuous-valued Logic}

The Dombi operator [2] in continuous-valued logic is given by

$$
o_{\alpha}\left(x_{1}, x_{2}, \ldots, x_{n}\right)=\frac{1}{1+\left(\sum_{i=1}^{n}\left(\frac{1-x_{i}}{x_{i}}\right)^{\alpha}\right)^{1 / \alpha}},
$$

where $x_{1}, x_{2}, \ldots, x_{n}$ are continuous-valued logic variables. If $\alpha \geq 0$, then the Dombi operator is a conjunction operator; if $\alpha \leq 0$, then the Dombi operator is a disjunction operator. The generator function $g_{\alpha}(x)$ of the Dombi operators in continuous-valued logic is given by

$g_{\alpha}(x)=\left(\frac{1-x}{x}\right)^{\alpha}$

Lemma 3. The generator function $g_{\alpha}(x)$ can be derived from the epsilon function $\varepsilon_{\lambda, d}(x)$ by a linear function transformation. 
Proof. Let us apply the $x^{\prime}=(x+d) /(2 d)$ linear transformation to the variable $x$, where $x \in(-d, d), d>0$. After this transformation, the domain of $x^{\prime}$ is the interval $(0,1), x=2 d x^{\prime}-d$, and

$$
\begin{aligned}
& \varepsilon_{\lambda, d}(x)=\left(\frac{x+d}{d-x}\right)^{\lambda \frac{d}{2}}=\left(\frac{2 d x^{\prime}-d+d}{d-2 d x^{\prime}+d}\right)^{\lambda \frac{d}{2}}=\left(\frac{x^{\prime}}{1-x^{\prime}}\right)^{\lambda \frac{d}{2}}= \\
& =\left(\frac{1-x^{\prime}}{x^{\prime}}\right)^{-\lambda \frac{d}{2}}=g_{\alpha}\left(x^{\prime}\right),
\end{aligned}
$$

where $\alpha=-\lambda d / 2$.

Based on this result, the generator function of the Dombi operators may be viewed as a special case of the epsilon function.

\section{Epsilon Probability Distribution}

Here, we will define the epsilon probability distribution and show how it is connected with the exponential distribution.

Definition 3. The continuous random variable $\xi$ has an epsilon probability distribution with the parameters $\lambda>0$ and $d>0$, if the probability density function $f_{\lambda, d}(x)$ of $\xi$ is given by

$f_{\lambda, d}(x)= \begin{cases}0, & \text { if } x \leq 0 \\ \lambda \frac{d^{2}}{d^{2}-x^{2}} \varepsilon_{-\lambda, d}(x), & \text { if } 0<x<d \\ 0, & \text { if } x \geq d,\end{cases}$

where

$\varepsilon_{-\lambda, d}(x)=\left(\frac{x+d}{d-x}\right)^{-\lambda \frac{d}{2}}$

In order to show that $f_{\lambda, d}(x)$ is in fact a probability density function, we will prove the following lemma.

Lemma 4. The function $f_{\lambda, d}(x)$ has the following properties.

1. $f_{\lambda, d}(x) \geq 0$ for any $x \in \mathbb{R}$

2. $\int_{-\infty}^{\infty} f_{\lambda, d}(x) \mathrm{d} x=1$.

Proof. The first property of $f_{\lambda, d}(x)$ trivially follows. The second property of $f_{\lambda, d}(x)$ can be demonstrated by using the definition of $\varepsilon_{-\lambda, d}(x)$ and the Lemma 2:

$\int_{-\infty}^{\infty} f_{\lambda, d}(x) \mathrm{d} x=\int_{0}^{d} \lambda \frac{d^{2}}{d^{2}-x^{2}} \varepsilon_{-\lambda, d}(x) \mathrm{d} x=\left[-\varepsilon_{-\lambda, d}(x)\right]_{0}^{d}=0-(-1)=1$. 
If the random variable $\eta$ has an exponential probability distribution with the parameter $\lambda>0$, then the probability density function $f_{\lambda}(x)$ of $\eta$ is given by

$f_{\lambda}(x)= \begin{cases}0, & \text { if } x \leq 0 \\ \lambda \mathrm{e}^{-\lambda x}, & \text { if } x>0\end{cases}$

The next theorem tells us how the epsilon probability distribution is connected with the exponential probability distribution.

Theorem 2. For any $x \in \mathbb{R}$ and $\lambda>0$, if $d \rightarrow \infty$, then

$f_{\lambda, d}(x) \rightarrow f_{\lambda}(x)$.

Proof. Let $x$ be fixed and let $x \in \mathbb{R}$. We will distinguish the following cases.

- If $x \leq 0$, then $f_{\lambda, d}(x)=f_{\lambda}(x)=0$ holds by definition.

- If $x \in(0, d), d>0$, then

$f_{\lambda, d}(x)=\lambda \frac{d^{2}}{d^{2}-x^{2}} \varepsilon_{-\lambda, d}(x)$.

If $d \rightarrow \infty$, then

$\frac{d^{2}}{d^{2}-x^{2}} \rightarrow 1$

and following Theorem 1,

$\varepsilon_{-\lambda, d}(x) \rightarrow \mathrm{e}^{-\lambda x}$.

That is, if $d \rightarrow \infty$, then

$f_{\lambda, d}(x) \rightarrow \lambda \mathrm{e}^{-\lambda x}=f_{\lambda}(x)$.

The probability distribution function $F_{\lambda, d}(x)$ of the random variable $\xi$ that has an epsilon probability distribution with parameters $\lambda>0$ and $d>0$ can be derived from the epsilon probability density function in the following way.

$$
\begin{aligned}
& F_{\lambda, d}(x)=\int_{-\infty}^{x} f_{\lambda, d}(t) \mathrm{d} t= \\
& = \begin{cases}\int_{-\infty}^{x} f_{\lambda, d}(t) \mathrm{d} t=\int_{-\infty}^{x} 0 \mathrm{~d} t=0, & \text { if } x \leq 0 \\
0 & \text { if } 0<x<d \\
\int_{-\infty}^{x} 0 \mathrm{~d} t+\int_{0}^{x} \lambda \frac{d^{2}}{d^{2}-t^{2}} \varepsilon_{-\lambda, d}(t) \mathrm{d} t, & \\
0 & d \\
\int_{-\infty}^{d} 0 \mathrm{~d} t+\int_{0}^{x} \lambda \frac{d^{2}}{d^{2}-t^{2}} \varepsilon_{-\lambda, d}(t)+\int_{d}^{x} 0 \mathrm{~d} t, & \text { if } x \geq d .\end{cases}
\end{aligned}
$$


As

$$
\begin{aligned}
& \int_{0}^{x} \lambda \frac{d^{2}}{d^{2}-t^{2}} \varepsilon_{-\lambda, d}(t) \mathrm{d} t=\left[-\varepsilon_{-\lambda, d}(t)\right]_{0}^{x}= \\
& =\left[-\left(\frac{t+d}{d-t}\right)^{-\lambda \frac{d}{2}}\right]_{0}^{x}=-\left(\frac{x+d}{d-x}\right)^{-\lambda \frac{d}{2}}-(-1)=1-\varepsilon_{-\lambda, d}(x)
\end{aligned}
$$

and

$\int_{0}^{d} \lambda \frac{d^{2}}{d^{2}-t^{2}} \varepsilon_{-\lambda, d}(t) \mathrm{d} t=1$,

$F_{\lambda, d}(x)$ may be written as

$F_{\lambda, d}(x)= \begin{cases}0, & \text { if } x \leq 0 \\ 1-\varepsilon_{-\lambda, d}(x), & \text { if } 0<x<d \\ 1, & \text { if } x \geq d .\end{cases}$

Note that if the random variable $\eta$ has an exponential probability distribution with the parameter $\lambda>0$, then the probability distribution function $F_{\lambda}(x)$ of $\eta$ is given by

$F_{\lambda}(x)= \begin{cases}0, & \text { if } x \leq 0 \\ 1-\mathrm{e}^{-\lambda x}, & \text { if } x>0 .\end{cases}$

Theorem 3. For any $x \in \mathbb{R}, \lambda>0$ and $d>0$, if the random variable $\xi$ has an epsilon probability distribution with the parameters $\lambda$ and $d$ and the $\eta$ random variable has an exponential distribution with parameter $\lambda$, then

$\lim _{d \rightarrow \infty} P(\xi<x)=P(\eta<x)$.

Proof. Since $F_{\lambda, d}(x)=P(\xi<x)$ and $F_{\lambda}(x)=P(\eta<x)$ for any $x \in \mathbb{R}$, using the definitions of $F_{\lambda, d}(x)$ and $F_{\lambda}(x)$, this theorem follows from Theorem 1.

Figure 2 shows some examples of how the density function curve of epsilon probability distribution can match the density function curve of exponential probability distribution. In each subplot of Figure 2, the left hand side scale belongs to functions $f_{\lambda}(x)$ and $f_{\lambda, d}(x)$, while the right hand side scale is connected with the difference function $f_{\lambda}(x)-f_{\lambda, d}(x)$. We can see that the goodness of approximation improves as $d$ increases. Similar to Figure 2, Figure 3 shows some examples of how the epsilon probability distribution function curve can match the exponential probability distribution function curve.

Based on Theorem 2, we may state that the asymptotic epsilon probability distribution is just the exponential probability distribution. Thus, in practical applications, the exponential probability distribution with a parameter $\lambda$ can be substituted by the 

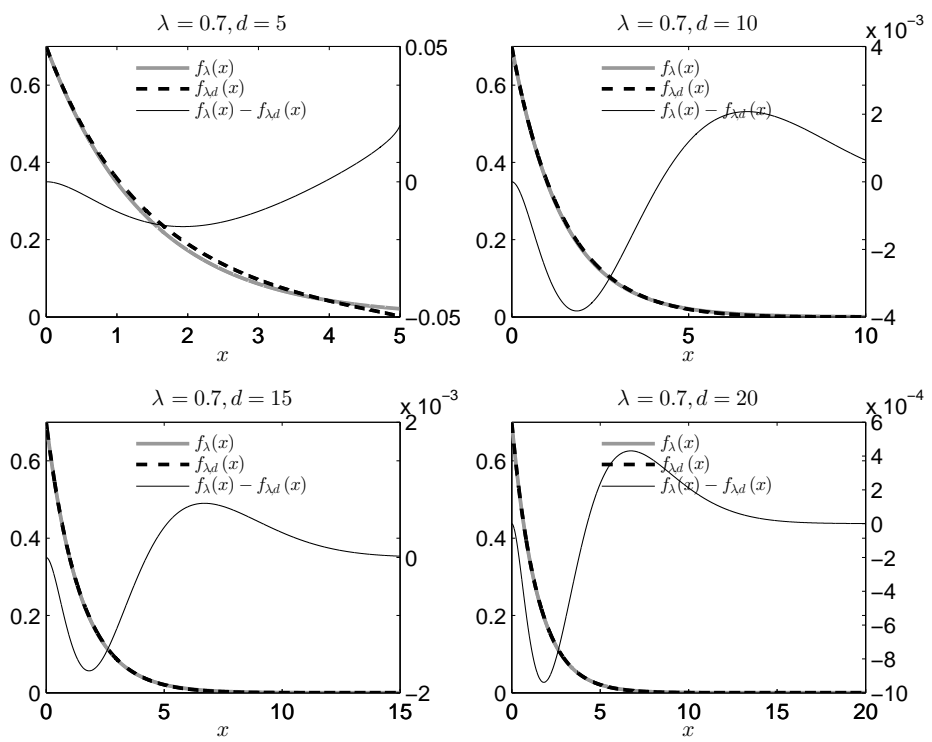

Figure 2

Examples of density function curves
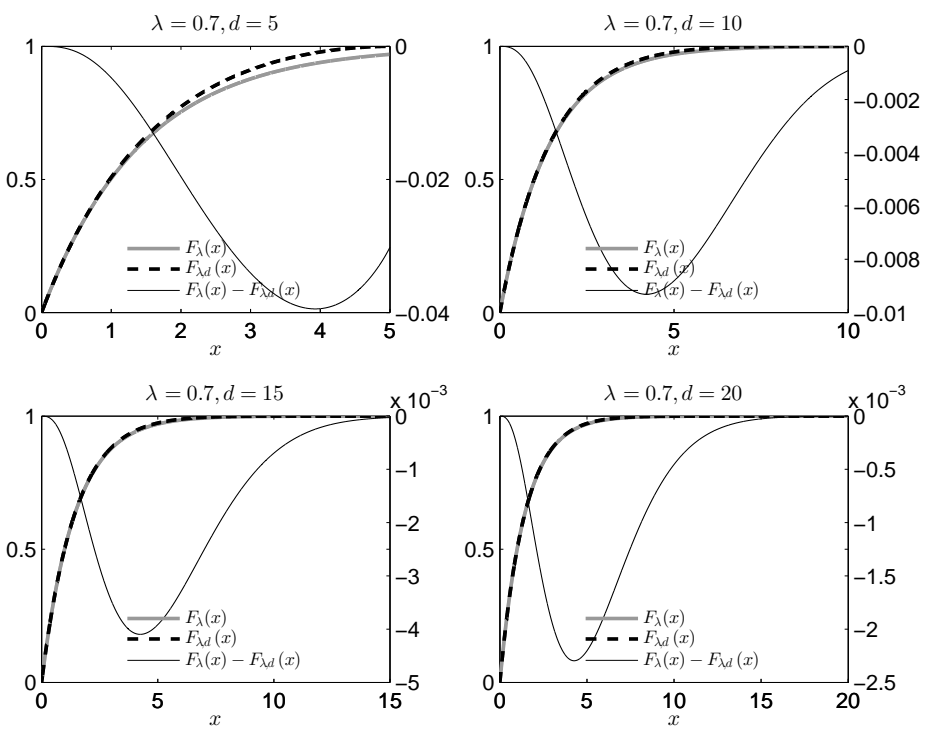

Figure 3

Examples of probability distribution function curves

epsilon probability distribution that has the parameters $\lambda$ and $d$, if $x \ll d$. It is worth mentioning that while the exponential probability distribution function is a transcendent function, the epsilon probability distribution function is a power function. This means that from a computational point of view, the epsilon probability distribution 
function is more convenient than the exponential probability distribution function. This feature of the epsilon probability distribution further enhances its applicability in problems where computation time is a critical factor.

From here on, we will use the notations $\xi \sim \varepsilon(\lambda, d)$ and $\eta \sim \exp (\lambda)$ to indicate that $\xi$ has an epsilon probability distribution with the parameters $\lambda>0$ and $d>0$, and $\eta$ has an exponential distribution with parameter $\lambda$, respectively.

\subsection{Some Asymptotic Properties of the Epsilon Probability Dis- tribution}

Here, we will show some asymptotic properties of the random variable $\xi$, where $\xi \sim \varepsilon(\lambda, d)$. Namely, we will show the asymptotic expected value and asymptotic standard deviation of $\xi$, and demonstrate the asymptotic memoryless property of $\xi$.

\section{Asymptotic expected value}

Theorem 4. If $\xi \sim \varepsilon(\lambda, d)$, then

$\lim _{d \rightarrow \infty} E(\xi)=\frac{1}{\lambda}$

where $E(\xi)$ is the expected value of $\xi$.

Proof. Here, using the definition of the expected value and Theorem 2

$$
\begin{aligned}
& \lim _{d \rightarrow \infty} E(\xi)=\lim _{d \rightarrow \infty} \int_{0}^{\infty} x f_{\lambda, d}(x) \mathrm{d} x= \\
& =\int_{0}^{\infty} x\left(\lim _{d \rightarrow \infty} f_{\lambda, d}(x)\right) \mathrm{d} x=\int_{0}^{\infty} x \lambda \mathrm{e}^{-\lambda x} \mathrm{~d} x .
\end{aligned}
$$

The last integral is the expected value of the random variable $\eta \sim \exp (\lambda)$. It is known that the expected value $E(\eta)$ of $\eta$ is $1 / \lambda$ and so

$$
\lim _{d \rightarrow \infty} E(\xi)=\frac{1}{\lambda}
$$

\section{Asymptotic standard deviation}

Theorem 5. If $\xi \sim \varepsilon(\lambda, d)$, then

$$
\lim _{d \rightarrow \infty} D(\xi)=\frac{1}{\lambda},
$$

where $D(\xi)$ is the standard deviation of $\xi$. 
Proof. Since $D^{2}(\xi)=E\left(\xi^{2}\right)-E^{2}(\xi)$,

$\lim _{d \rightarrow \infty} D^{2}(\xi)=\lim _{d \rightarrow \infty} E\left(\xi^{2}\right)-\lim _{d \rightarrow \infty} E^{2}(\xi)$.

Using Theorem 2,

$$
\begin{aligned}
& \lim _{d \rightarrow \infty} E\left(\xi^{2}\right)=\lim _{d \rightarrow \infty} \int_{0}^{\infty} x^{2} f_{\lambda, d}(x) \mathrm{d} x= \\
& =\int_{0}^{\infty} x^{2}\left(\lim _{d \rightarrow \infty} f_{\lambda, d}(x)\right) \mathrm{d} x=\int_{0}^{\infty} x^{2} \lambda \mathrm{e}^{-\lambda x} \mathrm{~d} x .
\end{aligned}
$$

The last integral can be calculated as follows.

$$
\begin{aligned}
& \int_{0}^{\infty} x^{2} \lambda \mathrm{e}^{-\lambda x} \mathrm{~d} x=\left[x^{2}\left(-\mathrm{e}^{-\lambda x}\right)\right]_{0}^{\infty}-\int_{0}^{\infty} 2 x\left(-\mathrm{e}^{-\lambda x}\right) \mathrm{d} x= \\
& =\frac{2}{\lambda} \int_{0}^{\infty} x \lambda \mathrm{e}^{-\lambda x} \mathrm{~d} x=\frac{2}{\lambda} \frac{1}{\lambda}=\frac{2}{\lambda^{2}} .
\end{aligned}
$$

Next, using Theorem 4,

$\lim _{d \rightarrow \infty} E^{2}(\xi)=\frac{1}{\lambda^{2}}$

and so

$\lim _{d \rightarrow \infty} D^{2}(\xi)=\lim _{d \rightarrow \infty} E\left(\xi^{2}\right)-\lim _{d \rightarrow \infty} E^{2}(\xi)=\frac{2}{\lambda^{2}}-\frac{1}{\lambda^{2}}=\frac{1}{\lambda^{2}}$,

from which after taking into account the fact that the $\lambda$ parameter always has a positive value, means that

$\lim _{d \rightarrow \infty} D(\xi)=\frac{1}{\lambda}$.

\section{Asymptotic memoryless property}

Definition 4. The random variable $\xi$ is memoryless with respect to t for all $\Delta t>0$, if

$P(\xi>t+\Delta t \mid \xi>t)=P(\xi>\Delta t)$.

It is known that the exponential distribution is the only continuous distribution that is memoryless. Here, we will show that $\xi \sim \varepsilon(\lambda, d)$ is an asymptotically memoryless random variable, if $d \rightarrow \infty$. 
Theorem 6. If $\xi \sim \varepsilon(\lambda, d)$, then

$\lim _{d \rightarrow \infty} P(\xi>t+\Delta t \mid \xi>t)=\lim _{d \rightarrow \infty} P(\xi>\Delta t)$.

Proof. Utilizing the definition of the conditional probability and the assumption that $\xi \sim \varepsilon(\lambda, d)$

$$
\begin{aligned}
& P(\xi>t+\Delta t \mid \xi>t)=\frac{P(\xi>t+\Delta t, \xi>t)}{P(\xi>t)}=\frac{P(\xi>t+\Delta t)}{P(\xi>t)}= \\
& =\frac{1-F_{\lambda, d}(t+\Delta t)}{1-F_{\lambda, d}(t)},
\end{aligned}
$$

and using (37)

$\frac{1-F_{\lambda, d}(t+\Delta t)}{1-F_{\lambda, d}(t)}=\frac{\varepsilon_{-\lambda, d}(t+\Delta t)}{\varepsilon_{-\lambda, d}(t)}$.

Furthermore, utilizing Theorem 1, equations (52) and (53),

$\lim _{d \rightarrow \infty} P(\xi>t+\Delta t \mid \xi>t)=\lim _{d \rightarrow \infty} \frac{\varepsilon_{-\lambda, d}(t+\Delta t)}{\varepsilon_{-\lambda, d}(t)}=\frac{\mathrm{e}^{-\lambda(t+\Delta t)}}{\mathrm{e}^{-\lambda t}}=\mathrm{e}^{-\lambda \Delta t}$.

Similarly, the right hand side of (51) may be written as

$\lim _{d \rightarrow \infty} P(\xi>\Delta t)=\lim _{d \rightarrow \infty} \varepsilon_{-\lambda, d}(\Delta t)=\mathrm{e}^{-\lambda \Delta t}$.

That is, both the left and right hand sides of (51) are equal to $\mathrm{e}^{-\lambda \Delta t}$.

\section{Application in Reliability Theory}

Let the continuous random variable $\tau$ be the time to first failure of a component or system. The conditional probability that this component or system will fail the first time in the time interval $(t, t+\Delta t]$, given that it has survived up to time $t$, can be calculated as follows:

$$
\begin{gathered}
P(\tau \leq t+\Delta t \mid \tau>t)=\frac{P(t<\tau \leq t+\Delta t)}{P(\tau>t)}= \\
=\frac{F(t+\Delta t)-F(t)}{1-F(t)}=\frac{F(t+\Delta t)-F(t)}{R(t)},
\end{gathered}
$$

where $F(t)$ is the probability distribution function of $\tau$ and $R(t)=1-F(t)$ is the survival function of $\tau$. In reliability theory, the failure rate function $h(t)$ for $\tau$ is given by

$h(t)=\lim _{\Delta t \rightarrow 0} \frac{F(t+\Delta t)-F(t)}{\Delta t R(t)}=\frac{f(t)}{R(t)}$, 
where $f(t)$ is the probability density function of $\tau$. The hazard function $h(t)$ is also called the failure rate function. In practice, based on (56) and (57), the quantity $h(t) \Delta t$ represents the conditional probability that a component or a system will fail in the time interval $(t, t+\Delta t]$, given that it has survived up to time $t$.

A typical hazard function curve of a component or a system is "bathtub shaped"; that is, it can be divided into three distinct phases called the infant mortality period, useful life, and wear-out period. During the early life (infant mortality), the failure rate function decreases with respect to time. In this phase, the failures are mostly caused by initial weaknesses or defects in the material, defective design, poor quality control, poor workmanship, and damaged or missing parts in the assembly phase. In the second phase, which is also known as the useful life, the failure rate function is constant with respect to time. In this period only random failures occur. These unexpected failures are caused by over-stress conditions and they cannot be eliminated by maintenance practices. Even the best design fabrication and screening techniques cannot completely eliminate the effect of such failures. Watson [21] gives reasons for the assumption of a constant failure rate. The third, wear-out phase can be described by an increasing failure rate function. The wear-out period can be postponed by introducing replacement technologies.

It is typical that the probability distribution of $\tau$ is different in the three characteristic phases of the "bathtub shaped" hazard function. If $\tau$ has an exponential distribution with parameter $\lambda$, then using (57), the hazard function $h_{\lambda}(t)$ for $\tau$ is

$h_{\lambda}(t)=\frac{f_{\lambda}(t)}{1-F_{\lambda}(t)}=\frac{\lambda \mathrm{e}^{-\lambda t}}{\mathrm{e}^{-\lambda t}}=\lambda$.

That is, if $\tau \sim \exp (\lambda)$, then the failure rate function is constant with respect to time. Most commonly, the exponential probability distribution is utilized to describe the probability distribution of $\tau$ in the second, constant phase of the hazard curve, while probability distributions that result in decreasing or increasing hazard functions are used to model the probability distribution of $\tau$ in the first and third phases of the hazard function curve.

Now let us assume that $\tau$ has an epsilon distribution with the parameters $\lambda$ and $d$. In this case, the hazard function $h_{\lambda, d}(t)$ is

$h_{\lambda, d}(t)=\frac{f_{\lambda, d}(t)}{1-F_{\lambda, d}(t)}=\frac{\lambda \frac{d^{2}}{d^{2}-t^{2}} \varepsilon_{-\lambda, d}(t)}{\varepsilon_{-\lambda, d}(t)}=\lambda \frac{d^{2}}{d^{2}-t^{2}}$,

if $0<t<d$. The hazard function $h_{\lambda, d}(t)$ has some important properties that are worth emphasizing here.

- If $t \in(0, d)$ is fixed, then $h_{\lambda, d}(t)$ tends to $\lambda$ as $d$ approaches infinity. In practice, it means that if $t$ is small compared to $d$, then $h_{\lambda, d}(t) \approx \lambda$. That is, if $d$ is sufficiently large, then the probability distribution of $\tau$ may be described by the epsilon distribution in the second phase of the hazard function.

- The hazard function $h_{\lambda, d}(t)$ is increasing with respect to $t$. If $t$ is small compared to $d$, then $h_{\lambda, d}(t)$ is increasing slowly, and $h_{\lambda, d}(t)$ tends to infinity as $t$ approaches $d$ (from the left hand side). 
Based on the above properties of the hazard function $h_{\lambda, d}(t)$, we may conclude that the epsilon probability distribution can be utilized to describe the probability distribution of the time to first failure random variable both in the second and in the third phases of the hazard function. Moreover, if the second phase of a failure rate function is slightly increasing instead of being constant with respect to time, then the probability distribution of $\tau$ in this phase of the hazard function can be better described by the epsilon probability distribution than by the exponential probability distribution.

\subsection{A practical example}

Now we will show how the epsilon probability distribution can be utilized to model the probability distribution of the time to first failure random variable in the second and third phases of the hazard function.

The failure rate function $h(t)$ can be estimated from empirical data by

$h(t) \approx \frac{N(t)-N(t+\Delta t)}{N(t) \Delta t}$,

where $N(t)$ is the number of components or systems that have survived up to time $t$ from the number of components or systems $N(0)$ that were initially put into operation. If $\Delta t=1$, then the estimated failure rate $h_{i}$ for period $i$ may be given by

$h_{i}=\frac{N(i)-N(i+1)}{N(i)}$,

$i=0,1, \ldots, n$, and so $h_{0}, h_{1}, \ldots, h_{n}$ may be viewed as an empirical failure rate time series.

Here, we examined the empirical failure rate times series corresponding to the second and third phases of the hazard function curve of an electronic product. The investigated time series, which is shown in Figure 4, contained 120 failure rates that had been computed from empirical data using Equation (61).

The hazard function $h_{\lambda, d}(t)$ was fitted to the first 30, 60, 90 and 120 data values of the empirical failure rate time series. In each case, the estimations $\lambda^{*}$ and $d^{*}$ of the parameters $\lambda$ and $d$, respectively, were identified by solving the following minimization problem:

$$
\begin{aligned}
& \mathscr{F}(\lambda, d)=\sum_{i=0}^{k}\left(h_{i}-\lambda \frac{d^{2}}{d^{2}-i^{2}}\right)^{2} \rightarrow \min \\
& \lambda>0, d>k
\end{aligned}
$$

We solved this minimization problem by applying an interior point algorithm [1]. In order to find the global minimum of the target function $\mathscr{F}(\lambda, d)$, the initial values for $\lambda_{0}$ and $d_{0}$ of $\lambda$ and $d$, respectively, were set as follows.

$$
\begin{aligned}
& \lambda_{0}=\frac{1}{k} \sum_{i=0}^{k} h_{i} \\
& d_{0}=k+\delta,
\end{aligned}
$$



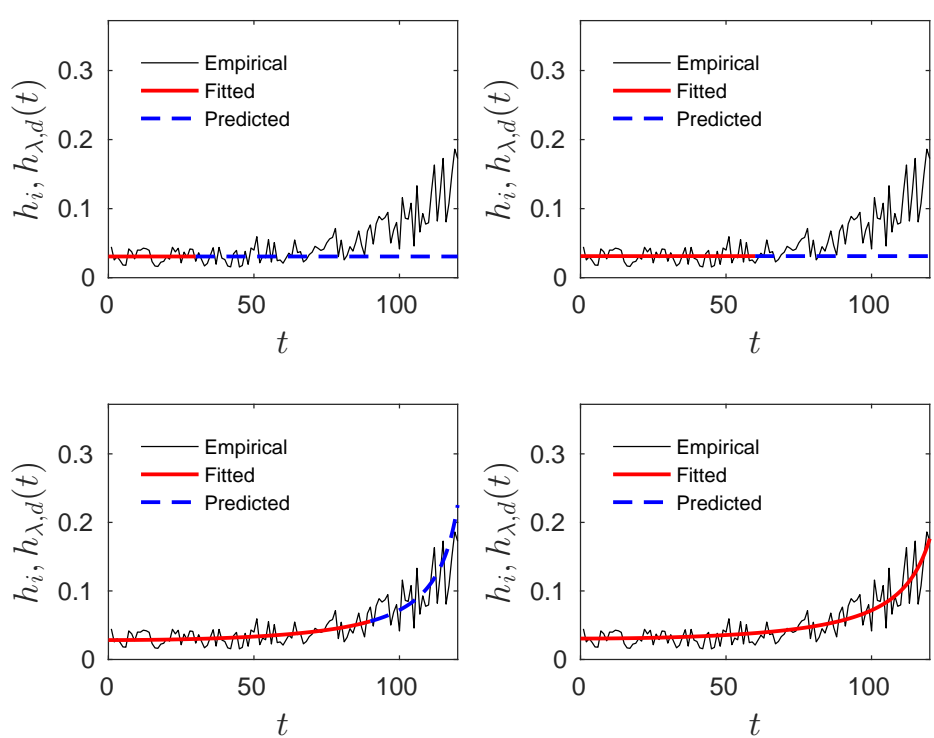

Figure 4

Hazard functions fitted to empirical failure rate time series

where $\delta>0$ is a number close to zero. In our implementation $\delta=10^{-3}$.

In each subplot of Figure 4, the continuous red line indicates the fitted $h_{\lambda, d}(t)$ values, while the dashed blue line shows the predicted failure rates that were based on the function $h_{\lambda, d}(t)$. Table 2 summarizes the mathematical modeling results. It contains the estimated model parameters and the Mean Square Error (MSE) values for the fits and predictions.

Table 2

Hazard function fitting results

\begin{tabular}{lllll} 
Input range & $\lambda^{*}$ & $d^{*}$ & MSE (Fitted) & MSE (Predicted) \\
\hline $0-29$ & 0.0307 & $1.8729 \cdot 10^{152}$ & $8.7506 \cdot 10^{-5}$ & 0.0022 \\
$0-59$ & 0.0312 & $3.1758 \cdot 10^{152}$ & $1.1538 \cdot 10^{-4}$ & 0.0032 \\
$0-89$ & 0.0283 & 128.3420 & $1.2907 \cdot 10^{-4}$ & 0.0010 \\
$0-119$ & 0.0305 & 131.7570 & $2.9315 \cdot 10^{-4}$ & \\
\hline
\end{tabular}

Based on Figure 4 and on Table 2, the following properties of our modeling should be mentioned here. The volatility of the analyzed failure rate time series increases with respect to time; that is, it displays a lower variability in its quasi constant phase than in its increasing phase. This observation is in line with the result that the MSE of the fitted curve slightly increases as the number of input data values increases. In the case of the first two input ranges, where the empirical hazard rate curve is in its quasi constant phase, the estimated values for the $d$ parameter are very large, while the estimates for the $\lambda$ parameter are very close. These results are in line with the previously discussed theoretical ones; namely, if $d$ is large and $t \ll d$, then the epsilon probability distribution is suitable for describing the time 
to first failure random variable in the quasi constant phase of the hazard function. When the empirical failure rate curve is in its increasing phase, then the estimates of parameter $d$ are close to the total length of the time series (120 periods), while the two estimates for the $\lambda$ parameter are similar. This empirical finding supports our theoretical results that the epsilon probability distribution can be utilized to describe the probability distribution of the time to first failure random variable in the third, increasing phase of the hazard function as well.

\section{Conclusions}

Here, we presented the epsilon probability distribution as a new distribution and suggested its possible applications in reliability theory and management. This new distribution is derived from the $n^{\text {th }}$ order epsilon differential equation. The solution of the zero order epsilon differential equation is the exponential, while the solution of the first order one is the epsilon function. Some basic properties including continuity, monotonity, limits and convexity were then stated. We also established an important connection between the exponential and the epsilon function; namely, the asymptotic epsilon function is just the exponential function. In practice it means that if $x \ll d$, then $\varepsilon_{\lambda, d} \approx \mathrm{e}^{\lambda x}$; that is, if $d$ is sufficiently large, then the epsilon function approximates the exponential function quite well. The revealed connection between the epsilon function and the Dombi operators in continuous-valued logic leads us to think that the generator function of the Dombi operators may be viewed as a special case of the epsilon function.

It should also be mentioned here that the epsilon probability distribution function is a power function when compared to the transcendent exponential probability distribution function. This feature is advantageous in cases where computation time is a competitive factor.

Focusing on the application of epsilon probability distribution in reliability management, this distribution can be utilized to describe the mortality and useful life period, assuming a typical bathtub-shaped failure rate. Our practical example also suggests that if the second phase of a failure rate function is slightly increasing rather than being constant with respect to time, the epsilon probability distribution will better describe the time to first failure random variable.

As for suggestions for possible future research, it might be interesting to examine the higher order $(n \geq 1)$ epsilon differential equations and their connections with other probability distributions.

\section{Acknowledgement}

This study was supported by Flextronics International Ltd, Budapest, Hungary.

\section{References}

[1] Waltz, R., Morales, J., Nocedal, J., Orban, D.,: An interior algorithm for nonlinear optimization that combines line search and trust region steps, Mathematical Programming, 2006, Vol. 9, pp. 391-408 
[2] Dombi, J.: General class of fuzzy operators, the demorgan class of fuzzy operators and fuzziness included by fuzzy operators, Fuzzy Sets and Systems, 1982, Vol. 8, pp. 149-168

[3] Yuge, T., Maruyama, M., Yanagi, S.: Reliability of a k-out-of-n system with common-cause failures using multivariate exponential distribution, Procedia Computer Science, 2016, Vol. 96, pp. 968-976

[4] Wang, L., Shi, Y.: Reliability analysis of a class of exponential distribution under record values, Journal of Computational and Applied Mathematics, 2013, Vol. 239, pp. 367-379

[5] Balakrishnan, N., Basu, A. P.: The exponential distribution. Theory, Methods and Applications. Gordon and Breach Publishers, Amsterdam, 1995

[6] Kondo, T.: Theory of sampling distribution of standard deviations, Biometrika, 1931, Vol. 22, pp. 31-64

[7] Steffensen, J. F.: Some recent research in the theory of statistics and actuarial science, Cambridge University Press, Cambridge, England, 1930

[8] Weibull, W.: The phenomenon of rupture in solids, Stockholm : Generalstabens Litografiska Anstalts Forlag, 1939

[9] Weibull, W.: A statistical distribution function of wide applicability, Journal of Applied Mechanics, 1951, Vol. 18, pp. 293-297

[10] Davis, D. J.: An analysis of some failure data, Journal of the American Statistical Association, 1952, Vol. 47, pp. 113-150

[11] Rossberg, H. J.: Über die Verteilungsfunktionen der Differenzen und Quotienten von Ranggrössen, Matematische Nachrichten, 1960, Vol. 21, pp. 37 79

[12] Rényi, A.: A characterization of the Poisson process, Magyar Tudományos Akadémia Matematika Kutató Intézet Közleménye, 1956, Vol. 1, pp. 519527 Translated into English in Selected papers of Alfréd Rényi, vol 1, Akadémiai Kiadó, 1976

[13] Ghurye, S. G.: Characterization of some location and scale parameter families of distributions, In Contributions to Probability and Statistics, 1960, pp. 202-215, Stanford University Press, Stanford, California

[14] Teicher, H.: Maximum likelihood characterization of distributions, Annals of Mathematical Statistics, 1961, Vol. 32, pp. 1214-1222

[15] Galambos, J., Kotz, S.: Characterizations of probability distributions, Lecture Notes in Mathematics, 1978, No. 675., Springer-Verlag, New York

[16] Mudholkar, G.S., Srivastava, D.K.: Exponentiated Weibull family for analyzing bathtub failure-rate data, IEEE Transactions on Reliability, 1993, Vol. 42, pp. 299-302 
[17] Rajarshi, S., Rajarshi M. B.: Bathtub distributions: A review, Communications in Statistics Theory and Methods, 1987, Vol. 17, No. 8, pp. 2597-2621

[18] Lai C.D., Xie, M., Murthy, D.N.P.: Bathtub-shaped failure rate life distributions, Handbook of Statistics, 2001, Vol.20, pp. 69-104

[19] Lemonte, A. J.: A new exponential-type distribution with constant, decreasing, increasing, upside-down bathtub and bathtub-shaped failure rate function, Computational Statistics and Data Analysis, 2013, Vol. 62, pp. 149-170

[20] Silva, R. B., Barreto-Souza, W., Cordeiro, G. M.: A new distribution with decreasing, increasing and upside-down bathtub failure rate, Computational Statistics and Data Analysis, 2010, Vol. 54, No. 4, pp. 935-944

[21] Watson, G. F.: MIL reliability: A new approach, IEEE Spectrum, 1992, Vol. 29, pp. 46-49

[22] O'Conor, P. D. T. (Ed.): Practical Reliability Engineering. Wiley, Chichester, England, 2006

NO PAGE NUMBERS 